\title{
Numerical Solution of Laminar Flow over Symmetric NACA Airfoils
}

\author{
Prasanna M.S.S ${ }^{1}$, Shashank Sadineni ${ }^{1}$, Rahul Kotikalapudi ${ }^{1}$, Dr. Prasad Pokkunuri ${ }^{1}$ \\ ${ }^{1}$ Department of Mechanical Engineering, Mahindra École Centrale, India \\ 1A, Survey No: 62, Bahadurpally, Hyderabad, India \\ sriprasanna170349@mechyd.ac.in; shashank17347@mechyd.ac.in
}

\begin{abstract}
Solving the Boundary Layer Equations is a challenge, even more so for complex geometries. This requires resolution of the drag inducing layer immediately adjacent to the solid surface, which is numerically and computationally intensive. Finite Difference schemes, though accurate, are better suited for rectilinear grids. The present work applies a unique approximation to solve the Boundary Layer Equations over a curved airfoil, approximating the geometry by linear splines, and sequentially applying the inclined flat plate solution over each individual section. The lift coefficient thus obtained for a NACA 0005 airfoil is compared with established values, for different angles of attack.
\end{abstract}

Keywords: Boundary Layers, Symmetric NACA Airfoils, Finite Difference Methods.

\section{Introduction}

Design and development of airfoils has always been a critical operation for the aviation industry. Aerodynamic analysis for airfoils, prior to the 1940s, was limited to 2D analytical methods using conformal transformations about a cylinder [1]. The earliest numerical methods are Finite Difference Schemes, pioneered by Richardson [2], and other potential flow based techniques. Naturally, the solutions obtained and the allowed complexity of the geometries to be optimised depended heavily on the computing power available. In parallel, the airfoils used in practice were of growing complexity and constantly evolved. In the 1930s, the National Advisory Committee for Aeronautics conducted studies on 'families' of foils and created the four-digit and five-digit series. Airfoil design finally moved from a manual, iterative procedure to mathematically precise analytical methods around the same time. NACA has since released multiple series of foils, each with its own characteristics. This study was further developed by Jacobs, who based the development of various profiles using pressure distribution arising from the boundary layer enveloping the foil. This kind of precision is fairly ubiquitous today, indicating the importance of the developing boundary layers [3]. First studied by Prandtl, Blasius and von Karmann [4], boundary layers indicate the region through which viscous effects are to be modelled. They were first solved analytically, and then numerically by the 1950s. The methods employed today rarely use the quintessential difference schemes, and eschew them in favour of more robust methods that can work with complex geometries faster. An important class of methods are the Panel methods, first outlined by Hess and Smith, 1967 [5]. These methods incorporate curved geometries using panels over the surface more easily than difference schemes, and are often faster. These methods have been extensively studied and developed over the years, growing in complexity, often using multi-order methods. As a tangent, this work considers a novel extension to the prototypical flat plate solution to solve for the steady state boundary layer of a symmetric NACA airfoil. The curved geometry of the airfoil is approximated as a series of inclined flat plates. Then, the boundary layer of each such plate is solved sequentially, where the velocity profile at the trailing edge of one plate is repurposed into the initial velocities for the next plate. The resulting velocity profiles give us the developed boundary layer.

The rest of the paper is organised as follows. The following sections detail the flat plate boundary layer, and then follow into the inclined flat plate problem. The latter is then extended to curved geometries, which we test on symmetric four-digit airfoils. The approximate solutions for the velocity fields are then used to compute the coefficient of lift, which is used to verify that the method does indeed model the boundary layer over the foil.

\section{Theory}

This section develops the flat plate boundary layer solution to solve the inclined flat plate problem. This solution is then used to construct the new method, which is used to solve boundary layer flow over curved geometries. 


\section{1. Boundary layer equations}

The governing equations for this flow are derived from the Navier-Stokes equations, which are as follows.

$$
\frac{\partial \mathbf{u}}{\partial t}+u \cdot \nabla \mathbf{u}=\frac{\Delta P}{\rho}+\nu \nabla^{2} \mathbf{u}
$$

where $\mathrm{u}$ is the horizontal component of the velocity field, and $\mathrm{v}$ is the vertical. Note that these are with respect to the global coordinate axes. When solving over each region, these will be resolved along appropriately inclined axes, and the meaning will be clear from context. $\mathbf{u}$ is the velocity field, with the aforementioned components. Lastly, $v$ is the kinematic viscosity of the fluid; in this case, air.

We are able to rewrite Eqn. (1) follows:

$$
\begin{gathered}
\frac{\partial u}{\partial t}+u \frac{\partial u}{\partial x}+v \frac{\partial u}{\partial y}=-\frac{1}{\rho} \frac{\partial P}{\partial x}+\nu \frac{\partial^{2} u}{\partial y^{2}} \\
\Longrightarrow u \frac{\partial u}{\partial x}+v \frac{\partial u}{\partial y}=\nu \frac{\partial^{2} u}{\partial y^{2}}
\end{gathered}
$$

subject to the following boundary conditions:

$$
\begin{gathered}
\text { At } y=0, x>0 ; u=v=0 \\
\text { At } y \rightarrow \infty, \forall x ; u=U_{\infty} \\
\text { At } x=0 ; u=U_{\infty}
\end{gathered}
$$

We are also specifying mass conservation throughout as follows.

$$
\Longrightarrow \frac{\nabla \cdot u=0}{\partial x}+\frac{\partial v}{\partial y}=0
$$

\section{2. Boundary Layer over a Flat Plate}

Consider a horizontal stream with velocity $U_{\infty}$ incident on a flat plate of negligible thickness as shown. Naturally, we are subject to the no-slip condition at the bottom of the plate, and we assume that we reach the free-stream velocity $U_{\infty}$ beyond the layer. These are essentially the conditions outlined in Eqn. 4.

Now, we can say that the problem is well posed. We can now begin discretising Eqn. 3. With help from (Blottner, 1970) [6], we use the following derivative approximations:

$$
\begin{aligned}
\frac{\partial u}{\partial x} & =\frac{U_{(i+1, j)}-U_{(i, j)}}{\Delta x} \\
\frac{\partial u}{\partial y} & =\frac{U_{(i, j+1)}-U_{(i, j)}}{\Delta y} \\
\text { and } \quad \frac{\partial^{2} u}{\partial y^{2}}= & \frac{U_{(i, j+1)}-2 U_{(i, j)}+U_{(i, j-1)}}{(\Delta y)^{2}}
\end{aligned}
$$

Here, $U_{(i, j)}$ denotes the steady flow velocity component at the $(i, j)^{t h}$ node. In accordance with the literature in Finite Difference Methods, each node represents a point in the domain where the velocity field is resolved. Naturally, $U_{(i+1, j)}$ refers to the $(i+1, j)^{t h}$ node i.e. the succeeding node along the (local) x-axis, and $U_{(i, j+1)}$ refers to the $(i, j+1)^{t h}$ node i.e. the succeeding node along the (local) y-axis. Note that because we deal with curved geometries, $\Delta x$ and , the node $\Delta y$ spacings along the $x$ and $y$ axes are variable. $(i, j)^{t h}$ node. In accordance with the literature in Finite Difference 
Methods, each node represents a point in the domain where the velocity field is resolved. Naturally, $U_{(i+1, j)}$ refers to the $(i+1, j)^{t h}$ node i.e. the succeeding node along the (local) $\mathrm{x}$-axis, and $U_{(i, j+1)}$ refers to the $(i, j+1)^{t h}$ node i.e. the succeeding node along the (local) y-axis. Note that because we deal with curved geometries, $\Delta x$ and, the node spacings along the $x \Delta y$ and $y$ axes are variable.

When Eqn. 6 is substituted in Eqn. 3, we get

$$
\begin{gathered}
U_{(i, j)} \frac{U_{(i+1, j)}-U_{(i, j)}}{\Delta x}+V_{(i, j)} \frac{U_{(i, j+1)}-U_{(i, j)}}{\Delta y} \\
=\nu \frac{U_{(i, j+1)}-2 U_{(i, j)}+U_{(i, j-1)}}{(\Delta y)^{2}}
\end{gathered}
$$

We can now rearrange this to form an equation in $U_{(i, j)}, \mathrm{U}_{(i, j-1)}$ and $U_{(i, j+1)}$, as follows.

$$
\begin{aligned}
& \quad\left(\frac{-\nu}{(\Delta y)^{2}}-\frac{V_{(i, j)}}{2 \Delta x}\right) U_{(i, j-1)}+\left(\frac{2 \nu}{(\Delta y)^{2}}+\frac{U_{(i, j)}}{\Delta x}\right) U_{(i, j)}+\left(\frac{-\nu}{(\Delta y)^{2}}-\frac{V_{(i, j)}}{2 \Delta x}\right) U_{(i, j+1)} \\
& \frac{U_{(i, j)}^{2}}{2 \Delta x}
\end{aligned}
$$

However, at the boundaries, we must modify our equation, which is then of the form

$$
\left(\frac{-\nu}{(\Delta y)^{2}}-\frac{V_{(i, j)}}{2 \Delta x}\right) U_{(i, j+1)}+\left(\frac{2 \nu}{(\Delta y)^{2}}+\frac{U_{(i, j)}}{\Delta x}\right) U_{(i, j)}=\left(\frac{-\nu}{(\Delta y)^{2}}-\frac{V_{(i, j)}}{2 \Delta x}\right) U_{(j-1, i+1)}
$$

near the plate, and

$$
\left(\frac{-\nu}{(\Delta y)^{2}}-\frac{V_{(i, j)}}{2 \Delta x}\right) U_{(i, j-1)}+\left(\frac{2 \nu}{(\Delta y)^{2}}+\frac{U_{(i, j)}}{\Delta x}\right) U_{(i, j)}=\left(\frac{-\nu}{(\Delta y)^{2}}-\frac{V_{(i, j)}}{2 \Delta x}\right) U_{(j+1, i+1)}
$$

near the edge of the domain. Now, we see that this method is implicit and thus can be resolved into a tridiagonal system to fully resolve the velocities at the $j^{\text {th }}$ layer. Because this is an implicit method, it is unconditionally stable; this allows us to have arbitrary levels of discretisation in the numerical analysis. $j^{\text {th }}$ layer. Because this is an implicit method, it is unconditionally stable; this allows us to have arbitrary levels of discretisation in the numerical analysis.

We study the case when $U_{\infty}=50 \mathrm{~m} / \mathrm{s}$, and $V_{0}=0$, i.e. a horizontal flow over a symmetric NACA 0005 foil. The fluid is assumed to be air, which gives us $\nu=1 \times 10^{-5}$, approximately. Further note that the foil is assumed to be smooth i.e. we neglect the wall-shear component in the following calculations. $U_{\infty}=50 \mathrm{~m} / \mathrm{s}$, and $V_{0}=0$, i.e. a horizontal flow over a symmetric NACA 0005 foil. The fluid is assumed to be air, which gives us $\nu=1 \times 10^{-5}$, approximately. Further note that the foil is assumed to be smooth i.e. we neglect the wall-shear component in the following calculations.

\section{3. Boundary layer over a symmetric airfoil}

Any airfoil is characterised by curved geometry, which is not easily amenable to an FD method. Past methods have tried to use meshed irregular grids i.e. grids with step size that varies with geometry or even tried to remove the mesh entirely Icite\{liszka1980finite $\}$. The latter is done via the random generation of nodes and using neighbouring nodes to compute the velocity. In any case, the fact remains that this difficulty in moving to complex geometries is one of the reasons for introducing more robust methods.

A key observation is that these methods depart significantly from the otherwise simple analysis outlined above. As engineers are wont to do, our method to compute the velocity field builds on the flat plate solution. We use linear splines to approximate the curved airfoil boundary as a series of flat plates, and then solve the flat plate problem over each region. The 
only added complexity is with the change in slope of each section, i.e. we now have to solve an inclined flat plate boundary flow problem.

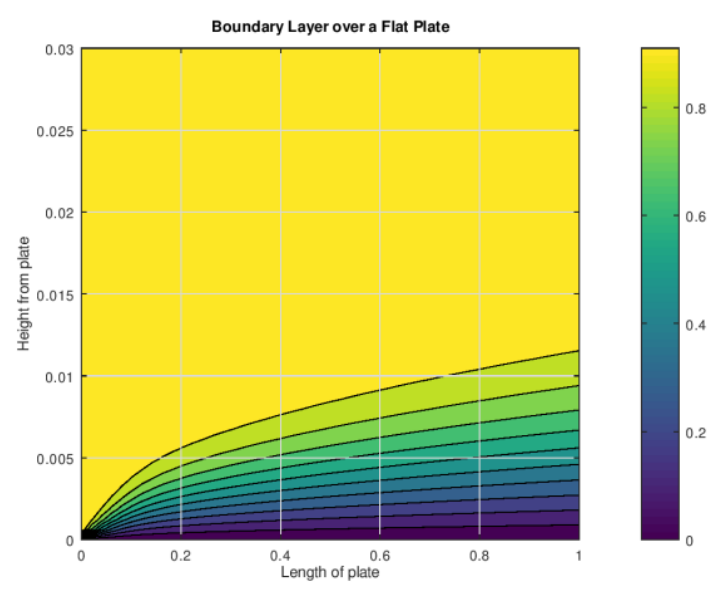

Fig. 1: Boundary layer over a flat plate. The x-axis is defined to be the surface of the plate, and the y-axis is the height from it in which the layer develops.

Solving an inclined flat plate presents only one difficulty over the normal problem. The incoming velocity field, though horizontal, will have to be resolved along the plate. Let us suppose that the velocity fields are essentially thus: $[U, V]^{T}=\left[U_{\infty}, 0\right]^{T}$, initially. Furthermore, let the slope of the plate be $\theta$. Then, denoting $\left[U^{\prime}, V^{\prime}\right]^{T}$ as the components as resolved on the plate, we can easily see that

$$
\left[\begin{array}{l}
U^{\prime} \\
V^{\prime}
\end{array}\right]=\left[\begin{array}{cc}
\cos (\theta) & \sin (\theta) \\
-\sin (\theta) & \cos (\theta)
\end{array}\right] \cdot\left[\begin{array}{c}
U_{\infty} \\
0
\end{array}\right]
$$

Note that this only resolves the velocities at the first plate i.e. at the leading edge. There are more spline interpolations along the curve. Fortunately, Eqn. leqref $\{$ resolve-velocity\} generalises easily to

$$
\left[\begin{array}{l}
U_{i} \\
V_{i}
\end{array}\right]=\left[\begin{array}{cc}
\cos \left(\theta_{i}\right) & \sin \left(\theta_{i}\right) \\
-\sin \left(\theta_{i}\right) & \cos \left(\theta_{i}\right)
\end{array}\right]\left[\begin{array}{l}
U_{i-1} \\
V_{i-1}
\end{array}\right]
$$

where $U_{i}$ denotes the incoming velocity profile at the $i^{\text {th }}$ plate and so on, and $\theta$ is the difference in the slopes between the $i^{t h}$ and $(i-1)^{t h}$ plates. With these modifications, we can repeatedly use the flat plate solution as outlined in Section 2.2.

Fig. 1 shows linear spline interpolations of airfoils with multiple thicknesses and zero camber, of which was the 5\% thickness profile was used to test this method. To reiterate, each segment of the spline will be treated as an inclined flat plate and solved thus. The velocity profiles at the ends of each plate/segment are stored as the velocities of the fluid along the foil. It is easily seen then, that a larger number of splines would result in a better approximation of the velocity profile.

\section{Results}

Using this solution, accuracy is compared with traditional methods. The accuracy of the method can be easily determined by a computation of the total lift force that the foil creates, as listed in Table 1. An approximate upper-bound calculation is used to find the lift coefficient, $C_{l}$, which is in close agreement with the theoretical values expected for the NACA 0005 foil used. This is listed as follows. 
Table 1. Approximate upper bound of lift coefficient vs actual lift coefficient in a NACA 0005 Airfoil with various angles of attack.

\begin{tabular}{|l|l|l|}
\hline $\begin{array}{l}\text { Angle of attack } \\
(\alpha)\end{array}$ & $\begin{array}{l}\text { Approx. Coefficient of } \\
\text { Lift }\left(C_{l}^{\prime}\right)\end{array}$ & $\begin{array}{l}\text { Theoretical Coefficient } \\
\text { of Lift }\left(C_{l}\right)\end{array}$ \\
\hline $2^{\circ}$ & 0.24 & 0.219 \\
\hline $4^{\circ}$ & 0.43 & 0.438 \\
\hline $6^{\circ}$ & 0.67 & 0.657 \\
\hline $8^{\circ}$ & 0.76 & 0.87 \\
\hline $10^{\circ}$ & 0.80 & 0.6 \\
\hline
\end{tabular}

Importantly, the drag force caused by the foil is an extremely important factor in airfoil design, and so, an easily implementable solution with a good degree of accuracy is important. Finally, the velocity field as resolved along the geometry is also plotted, in Figure 2(a). This shows the resolution and development of the boundary layer over the airfoil.

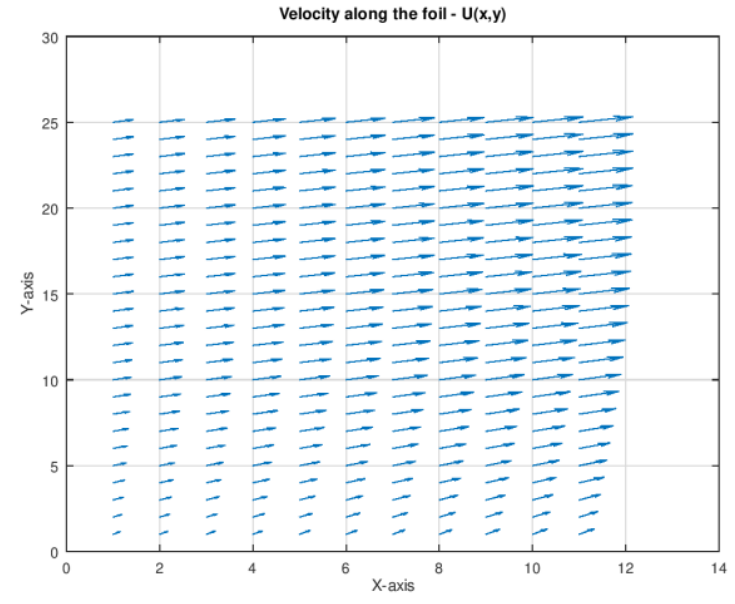

(a)

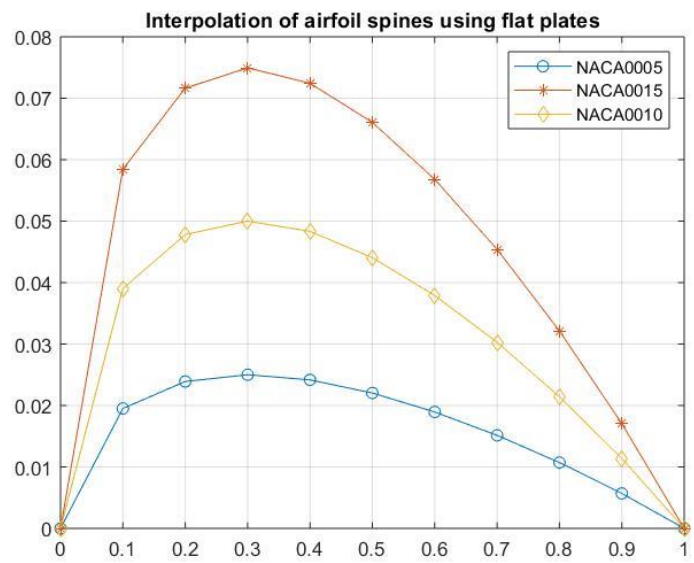

(b)

Fig. 2: Plotting the resulting velocity field to observe the developing boundary layer. (a) Velocity field $\vec{u}(x, y)$ over the domain. (b) The linear spline approximation, used for foils of 5\%,10\% and $15 \%$ thicknesses with no camber.

\section{Conclusion}

The inclined flat plate boundary layer solution is sequentially applied, piecewise, to approximate the flow-field over a curved airfoil. The lift coefficient is obtained from the flow-field so calculated, and values obtained closely match well established results. The deviation, however, increases at higher angles of attack, owing to inherent simplifications in the method. Furthering the work done so far, the authors propose to quantify approximation errors, which can then be used to extend the method to more complex geometries such as cambered airfoils, and at larger angles of attack.

\section{Appendix: Calculating Lift Coefficient}

The coefficient of lift has to be calculated using the velocity profiles over each individual plate. Note that the coefficient of lift is calculated as follows. To find the lift force $L$, note that for an individual segment of length $\Delta l$, 


$$
\Delta L=\Delta P \cdot A=\frac{1}{2} \rho\left(V_{u}^{2}-V_{l}^{2}\right)(b \cdot d l)
$$

where $V_{u}^{2}$ denotes the boundary layer velocity when developed over the upper segment of the foil, $V_{l}$ is the same velocity developed on the lower segment, and $b$ is the depth of the plate. For simplicity, we ignore any fringe effects. Now, we can sum over all the plates to get

$$
L=\sum_{i} \Delta L_{i}=\frac{1}{2} \rho \sum_{i}\left(V_{u, i}^{2}-V_{l, i}^{2}\right)\left(b \cdot d l_{i}\right)
$$

Therefore, we can use the definition of $C_{l}$ as follows.

$$
\begin{aligned}
C_{l}= & \frac{\frac{1}{2} \rho \sum_{i}\left(V_{u, i}^{2}-V_{l, i}^{2}\right)\left(b \cdot d l_{i}\right)}{\sum_{2} \frac{1}{2} \rho\left(b \cdot d l_{i}\right) U_{\infty}^{2}}=\frac{\sum_{i}\left(V_{u, i}^{2}-V_{l, i}^{2}\right) \cdot d l_{i}}{\sum_{\infty} d l_{i} U_{\infty}^{2}} \\
& \leq \frac{\sum_{i}\left(V_{u, i}^{2}-V_{l, i}^{2}\right) \cdot \sum_{i} d l_{i}}{\sum d l_{i} U_{\infty}^{2}} \leq \frac{\sum_{i}\left(V_{u, i}^{2}-V_{l, i}^{2}\right)}{U_{\infty}^{2}}
\end{aligned}
$$

Finally, assuming the mean velocities $\bar{V}_{u}$ and $\$ \operatorname{lbar}\left\{\mathrm{V}_{-} 1\right\} \bar{V}_{\mathrm{ar}}\left\{\mathrm{V} \_1\right\} \$$, we get

$$
C_{l} \leq n \frac{\left(\bar{V}_{u}^{2}-\bar{V}_{l}^{2}\right)}{U_{\infty}^{2}}
$$

where $i \in[n]$, for some $n \in \mathbb{N}$. Using the approximation detailed above, we obtain the computed values of $C_{l}$ as shown in Tab. 1. Their close agreement allows us to see that the method developed does indeed model the foil accurately. The theoretical values are derived from Eqn. from Thin Airfoil theory.

$$
C_{L}=2 \pi \alpha
$$

where $\alpha$ is the angle of attack, and $C_{L}$ is the lift coefficient.

\section{References}

[1] L. M. Milne-Thomson, Theoretical aerodynamics. Courier Corporation, 1973.

[2] W. Covey, "Weather prediction by numerical process: By lewis f. richardson, dover publications, inc. paperback. 1965.," Soil Science Society of America Journal, vol. 30, no. 1, pp. vi-vi, 1966.

[3] E. N. Jacobs, K. E. Ward, and R. M. Pinkerton, The Characteristics of 78 Related Airfoil Section from Tests in the Variable-Density Wind Tunnel. No. 460, US Government Printing Office, 1933.

[4] T. Liszka and J. Orkisz, "The finite difference method at arbitrary irregular grids and its application in applied mechanics," Computers and Structures, vol. 11, no. 1-2, pp. 83-95, 1980.

[5] J. L. Hess and A. Smith, "Calculation of potential flow about arbitrary bodies," Progress in Aerospace Sciences, vol. 8, pp. 1-138, 1967.

[6] F. Blottner, "Finite difference methods of solution of the boundary-layer equations," AIAA journal, vol. 8, no. 2, pp. 193-205, 1970. 